\title{
Impant placement on a Focal Cemento-Osseous Dysplasia : A modified protocol with a successful outcome.
}

\author{
Mootaz MLOUKA ${ }^{1}$, Mohamed Tlili ${ }^{1}$, Faten Khanfir $^{1}$, ali meddeb hamrouni ${ }^{1}$, Mohamed \\ Salah Khalfi ${ }^{1}$, and Faten Ben Amor ${ }^{1}$ \\ ${ }^{1}$ University of Monastir Faculty of Dental Medicine of Monastir
}

November 23, 2021

\begin{abstract}
Cemento-Osseous dysplasia(COD) is a condition where normal bone is replaced by fibrous connective tissue and cementum-like deposits. Implant rehabilitation of a posterior mandibular edentulism in presence of a COD can be a challenging situation due to the lack of vascularization and the high risk of infection of such lesions.
\end{abstract}

Title : Impant placement in a Focal Cemento-Osseous Dysplasia : A modified protocol with a successful outcome.

Short Title : Focal Cemento-Osseous Dysplasia and implant therapy : A case report.

Authors : M.Mlouka, M. Tlili, F. Khanfir, A. Hamrouni, M.S Khalfi, F. Ben Amor

Abstract :

Cemento-Osseous dysplasia (COD) is defined as a condition in which normal bone is replaced by fibrous connective tissue and cementum-like deposits. It's generally asymptomatic, and occurs mostly in female patients. Radiologically, it appears as an opaque, lobulated mass.

When facing such lesions, it is advised to avoid performing any surgical procedures due to the decreased vascularization and healing potential. The main reported complications being poor healing, sequestrum formation, risk of infection and fracture of the jaw.

treating posterior mandibular edentulism in presence of a COD can be a challenging situation especially when the patient requires an implant-supported fixed rehabilitation.

The aim of this paper is to describe a three-stage modified protocol for implant placement in a compromised site presenting a Focal Cemento-Osseous Dysplasia.

Introduction :

Cemento-Osseous Dysplasia is a benign fibro-osseous lesion affecting mainly African / African American female patients aged between 40 and 50 (1).

COD is classified into 3 categories according to its location;

- Periapical COD in the periapical region of the anterior teeth.

- Focal COD associated with a single tooth.

- Florid COD (FCOD). In FCOD, lesions appear in more than 1 quadrant predomintly in the mandible (2). 
Radiologically, the lesion is radiolucent at first but becomes progressively opaque as it matures with the deposition of a cementum-like non-vascularized tissue (3). For this reason, elective surgery such as periodontal surgery, extractions or implant placement is contraindicated (4).

In this case report, we will present a successful implant rehabilitation of a posterior mandibular edentulism in a patient presenting focal COD using a modified protocol.

Case presentation :

A 48-year old female patient was referred to the Outpatient and Implantology department of the University Dental Clinic of Monastir - Tunisia for an implant rehabilitation. She was non-smoker and the medical history did not reveal any significant systemic diseases.

Clinically, she presented two edentulous sites ; the first one was located in the right maxilla (tooth 16 and 17), while the second was located in the right mandible (tooth 47). Only the management of the mandibular site will be described in this paper.

A dental panoramic radiograph $(\mathrm{OPG})$ was prescribed and showed a radiopaque lobular lesion in the site of tooth 47. Later, the CBCT's coronal sections enabled a better vizualisation of the lesion. The findings of the radiological examination were attributable to focal COD. (Figure 1, Figure 2).

The patient was informed of all the prosthetic solutions and the possible complications, and still requested an implant rehabilitation. An informed consent was signed prior to the procedure.

The implant surgery was performed in 3 steps to minimize the risk of infection :

- 1st stage: After local anesthesia, a crestal incision completed with 2 partial intra-sulcular incisions on the adjacent teeth were performed. Then, a full-thickness flap was elevated and the drilling sequence was carried out. Afterwards, tooth 48 was extracted because of dental decay and the flap was repositioned and sutured. (Figures $3,4,5,6,7,8)$.

Despite the prescription of an association of antibiotics (amoxicillin-clavulanic acid) after the first surgery, the 7-day check-up revealed signs of infection such as pain and local swelling. The abscess was drained and a betadine wash was performed to control the infection. (Figure 9).

- 2nd stage : 3 weeks afterwards: the flap was re-opened, an implant (4.2 mm x 10 mm, Kontactß, Biotech dental, France) was placed into the prepared site and the cover screw was put in place. Finally, the flap was sutured. (Figures 10, 11, 12).

- 3rd stage : 3 months after the 2nd stage : the cover screw was replaced by the healing abutement. (Figure $13)$.

Discussion :

Cemento-Osseous Dysplasia (COD) :

COD is a fibro-osseous lesion caracterized by a modification of the bone sturcture. Histologically, it appears as a cellular fibrous stroma with mineralizing tissues consisting of osteoid, bone and cementum-like material. The diagnosis requires a combined assessment of clinical and radiographic findings (5). In its late stages, the lesion appears as a radiopaque lobulated calcified mass surrounded by a radiolucent margin (4). The pathogenesis remains unknown.

The classification and terminology of these lesions is controversial and has drastically evolved over the years (6). The latest World Health Organization (WHO) classification published in 2017 defined three categories of COD based on their anatomic location. In fact, COD lesions have periapical, focal, and florid variants.

Periapical-COD (PCOD) mainly involves the periapical region of the anterior mandibular teeth, whereas focal-COD (FocCOD) is usually located in the mandibular molar region, often in edentulous areas. Finally 
the florid-COD (FCOD) has more specific and distinct clinical and radiographic signs compared to the other two variants: It involves two or more jaw quadrants and is in most cases bilateral and symmetric (7).

COD mostly affects the middle-aged African / African-American women. In fact, A systematic review published in 2003 assessed 159 cases of FCOD ; 59\% occurred in blacks, 37\% in Asians, and 3\% in Caucasians; $97 \%$ of all patients were female (8).

\section{COD and implant therapy :}

In the presence of a COD, the general rule is to avoid any type of elective surgery such as extractions, periodontal surgery or implant therapy (4). Implant placement in these cases should only be performed after the patient has been informed of the risks and signed a consent form.

Litterature treating the subject of implant rehabilitation with COD is quite poor. It has been suggested that implant placement in an affected site could induce osteomyelitis. In fact, overheating during the drilling sequence, added to the lack of vascularization and the reduced capacity of bone regeneration would lead to infection and necrosis of the surrounding tissue (9). Moreover, the lesion's response to antibiotics would be insufficient due to its avascular nature (3).

Shin et al. reported a case of chronic osteomyelitis induced by the placement of dental implants on COD. The implants failed to osseointegrate and were removed along with a sequestrum after an episode of swelling and pain (10).

Nevertheless, Park et al. described a case of successful osseotegration of an implant placed in a dysplasic site. The implant survived 15 years without any complication. However, at the 16-year follow-up, the implant was removed with a sclerotic mass due to peri-implantatis. Histology showed that the hard mass attached to the implant was formed by a cementum-like tissue (CLT) free of any soft tissue. The implant placed into the FCOD lesion achieved integration similar to that of conventional osseointegration. Rather than bone, the CLT was in direct contact with the titanium surface. The integrity of the union was maintained for up to 16 years $(11)$.

To our knowledge, two case reports have described a successful implant rehabitlitation on COD. Shadid et al. reported a successful implant rehabilitation of a lower posterior edentulous site in presence of a FCOD. 2 implants were placed with strict infection control. This was acheived by prescribing prophylactic antibiotic (amoxicillin/clavulanic acid $500 \mathrm{mg} / 125 \mathrm{mg}$ ) $1 \mathrm{~h}$ before surgery and chlorhexidine mouthwash preoperatively and postoperatively, by ensuring sterile environment during the procedure, minimizing periosteal reflection, making the procedure as short as possible, and avoiding overheating of the bone using cool sterile saline and sharp drills. No complications have occured after an 8-year follow-up (12).

Similarly, another case report described a successful implant rehabilitation of a posterior lower edntulism with an uneventful functional integration at the 18-month follow-up (13).

The proposed three-stage protocol:

The protocol used in our case report consists in deviding implant placement into 3 distinct surgeries :

- First stage : Drilling sequence with abundant cooling solution, betadine rinsing of the newly-created socket, hermetic closure of the wound and prescription of antiobiotic therapy.

- Second stage : Reopening of the site after 3 weeks (proliferation phase of the socket healing process) and implant placement.

- Third stage : Replacing the cover screw with the healing abutement after a healing period of 3 months.

The frist surgery is undoubtedly the most risky ; in fact, drilling through the dysplasic tissue generates heat which increases the risk of infection and necrosis of the surrounding tissue. Systematic antiotherapy is highly advised in this stage to prevent such risks. In case it proved unsufficient, infection must be treated with surgical curettage of the site, betadine rinsing and the prescription of an antiseptic mouthwash. 
Delaying implant insertion 3 to 4 weeks comes with 2 major advantages ; First, preventing implant surface contamination in case of infection due to the drilling sequence. And second, the time lapse between the drilling sequence and implant placement matches the proliferation phase of the socket healing process caracterized by the formation of a woven immature bone surrounded by a provisional fibrous matrix (fig 14)(14). It has been hypothesized that this newly-created healthy tissue surroundig the implant would act more like normal bone and would be more compatible with implant osseointegration than the cementum-like tissue originally found in COD.

In addition, delaying the placement of the healing abutement is highly advised. In fact, exposing the implant to the oral cavity's flora simultaniously with its insertion increases the risk on infection. A 3-month delay enables the surrounding hard tissue to reach a more mature state corresponding to the remodeling phase of the socket healing process (14).

\section{Conclusion :}

Placing an implant in a site with COD comes with a high risk on infection. Patients must be informed of all the therapeutic options and a consent form must be signed beforehand.

If implant rehabilitation is requested, our main concern is to minimize the risk of infection especially during and after the drilling sequence. The three-stage protocol described in this paper proved to be successful in terms of preventing contamination of the implant surface even when infection occurs. Further clnical and histological studies must be carried out to further assess the benefits of this protocol.

\section{Conflict of Interests}

The authors declare that there is no conflict of interests regarding the publication of this paper.

\section{Ethical Statement:}

This manuscript is the authors' own original work, which has not been previously published or considered for publication elsewhere.

The paper properly credits the meaningful contributions of co-authors and co-researchers. All authors have been personally and actively involved in substantial work leading to the paper, and will take public responsibility for its content.

\section{funding}

this paper did not receive any special funding.

Authors' details:

Author 1 : Mootaz Mlouka ${ }^{1}$ : ensured patient follow-up and manuscript redaction.

Author 2 : Mohamed Tlili $^{2}$ : surgery performance and manuscript revision.

Author 3 : Faten Khanfir ${ }^{3}$ : manuscript revision.

Author 4 : Ali Hamrouni ${ }^{1}$ : manuscript drafting.

Author 5 : Mohamed Salah Khalfi ${ }^{3}$ : manuscript revison.

Author 6 : Faten Ben Amor ${ }^{3}$ : manuscript revision.

${ }^{1}$ Resident in Anatomy and Oral Surgery

${ }^{2}$ Assistant professor in Anatomy and Oral Surgery

3 Professor in Anatomy

University/Resarch Unit: 
University of Monastir, Faculty of Dental Medicine, Research Laboratory of Oral Health and Oro Facial Rehabilitation, LR12ES11, 5000, Monastir, Tunisia;

References :

1. Cyntia Helena Pereira et al Florid cemento-osseous dysplasia and osteomyelitis: A case report of a simultaneous presentation. Rev OdontoCienc 2012

2. Cavalcanti PH, Nascimento EH, Pontual ML, Pontual AD, Marcelos PG, Perez DE, et al. Cementoosseous dysplasias: imaging features based on cone beam computed tomography scans. Braz Dent J. 2018;29:99-104.

3. Bencharit S, Schardt-Sacco D, Zuniga JR, Minsley GE. Surgical and prosthodontic rehabilitation for a patient with aggressive florid cemento-osseous dysplasia: a clinical report. J Prosthet Dent. 2003 Sep;90(3):220-4.

4. Gerlach RC, Dixon DR, Goksel T, Castle JT, Henry WA. Case presentation of florid cemento-osseous dysplasia with concomitant cemento-ossifying fibroma discovered during implant explantation. Oral Surg Oral Med Oral Pathol Oral Radiol. 2013 Mar;115(3):e44-52.

5. El-Mofty SK. Fibro-osseous lesions of the craniofacial skeleton: an update. Head Neck Pathol 2014;8:432-44

6. Noffke CE, Raubenheimer EJ, MacDonald D. Fibro-osseous disease: harmonizing terminology with biology Oral Surg Oral Med Oral Pathol Oral Radiol 2012;114:388-92

7. El-Mofty SK, Nelson B, Toyosawa S, Wright J. Fibro-osseous and osteochondromatous lesions. ElNaggar, Chan JKC, Grandis JR, Takata T, Slootweg PJ, editors. WHO classification of Head and Neck Tumours. 4th ed. Lyon:IARC, 2017, p. 251-55

8. MacDonald-Jankowski DS. Florid cemento-osseous dysplasia: a systematic review. Dentomaxillofac Radiol. 2003;32:141-9.

9. Shnaiderman-Shapiro A, Dayan D, Buchner A, Schwartz I, Yahalom R, Vered M. Histopathological spectrum of bone lesions associated with dental implant failure: osteomyelitis and beyond. Head Neck Pathol. 2015 Mar;9(1):140-6.

10. Shin HS, Kim BC, Lim HJ, Jo SY, Lee J. Chronic osteomyelitis induced by the placement of dental implants on cemento-osseous dysplasia. Br J Oral Maxillofac Surg. 2019 Apr;57(3):268-270.

11. Park WB, Han JY, Jang JS, et al. Longterm implant survivability of an implant having direct contact with cementumlike tissue in a preexisting mandibular intraosseous lesion with a 16-year longitudinal follow-up. Int J Periodontics Restorative Dent. 2019 Nov/Dec;39(6):895-902.

12. Shadid R, Kujan O. Success of dental implant osseointegration in a florid cemento-osseous dysplasia: A case report with 8-year follow-up. Clin Pract. 2020 Sep 4;10(3):1281

13. Esfahanizadeh N, Yousefi H. Successful Implant Placement in a Case of Florid Cemento-Osseous Dysplasia: A Case Report and Literature Review. J Oral Implantol. 2018 Aug;44(4):275-279.

14. de Sousa Gomes P, Daugela P, Poskevicius L, Mariano L, Fernandes MH. Molecular and Cellular Aspects of Socket Healing in the Absence and Presence of Graft Materials and Autologous Platelet Concentrates: a Focused Review. J Oral Maxillofac Res. 2019 Sep 5;10(3):e2.

Figure legends :

Figure 1 : Pre-operative panoramic reconstruction.

Figure 2 : CBCT coronal sections of the site of tooth 47 .

Figure 3 : Full-thickness flap elevation.

Figure 4 : Drilling of the implant site.

Figure 5 : Use of a parallel guide to verify the axis.

Figure 6 : Betadine wash.

Figure 7 : Extraction of tooth 48 and sutures. 
Figure 8 : Post-operative retroalveolar radiography.

Figure 9 : infection of the site (7 day follow-up).

Figure 10 : Elevation of a full-thickness flap.

Figure 11 : Implant placement.

Figure 12 : post-operative radiography.

Figure 13 : Placement of the healing abutement.

Figure 14 : The socket healing process.





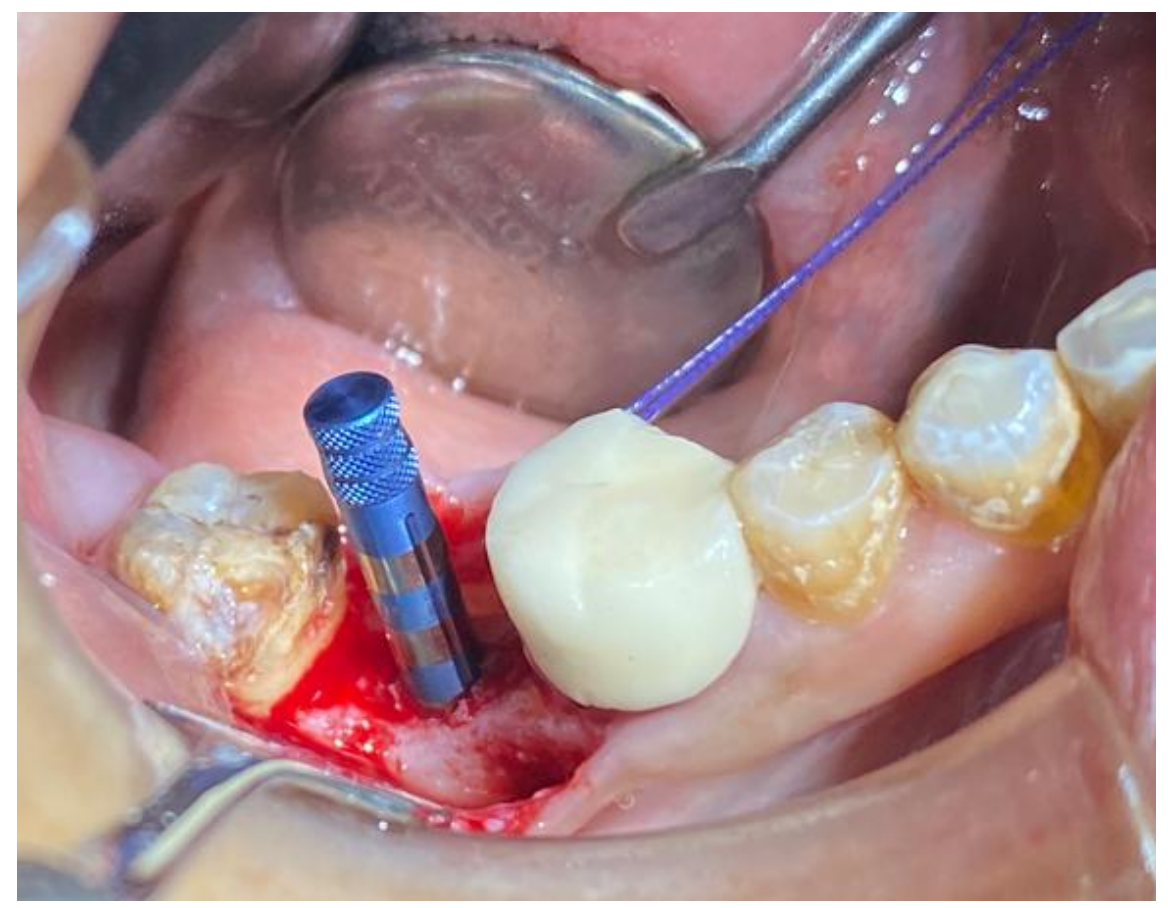




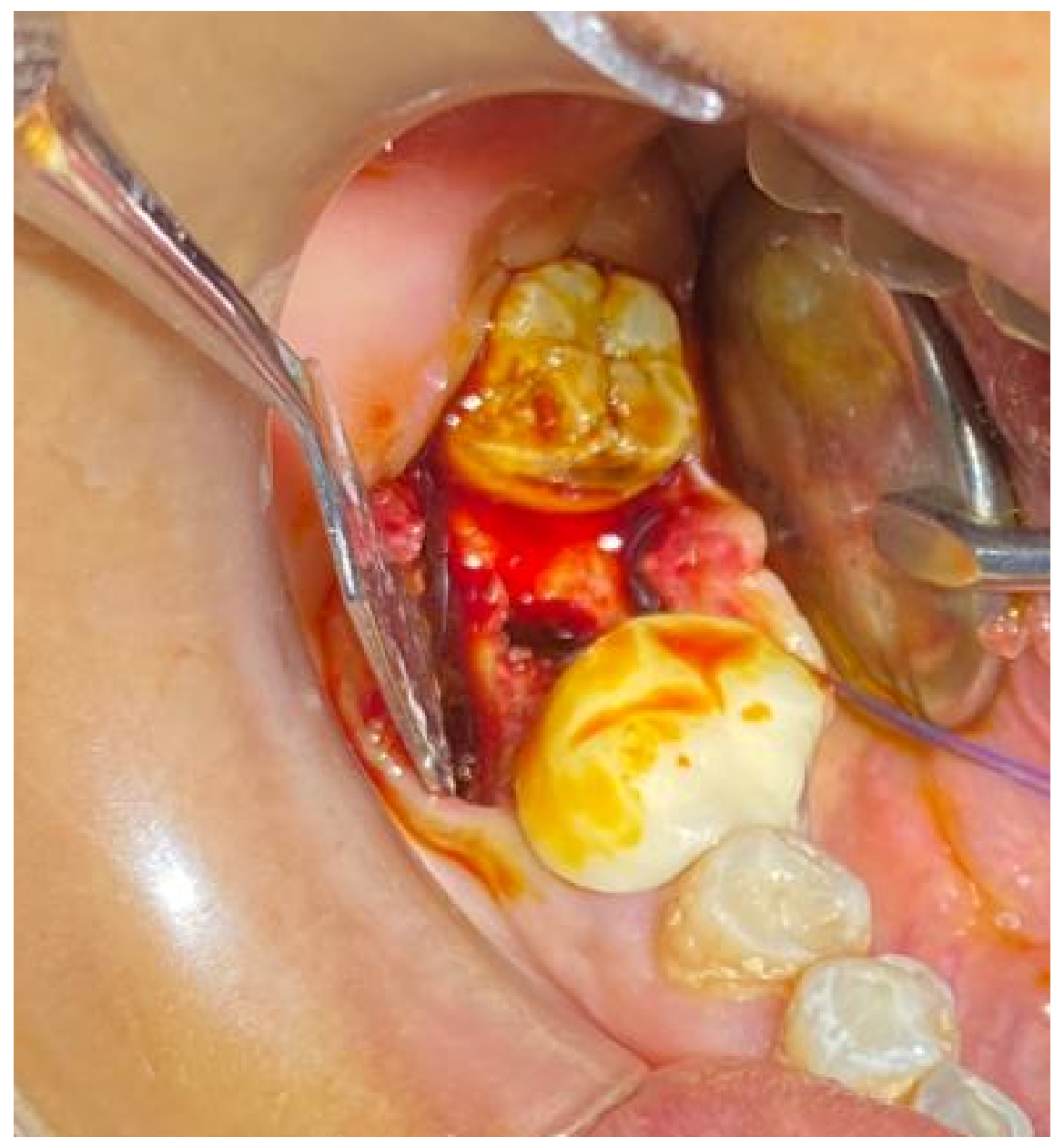










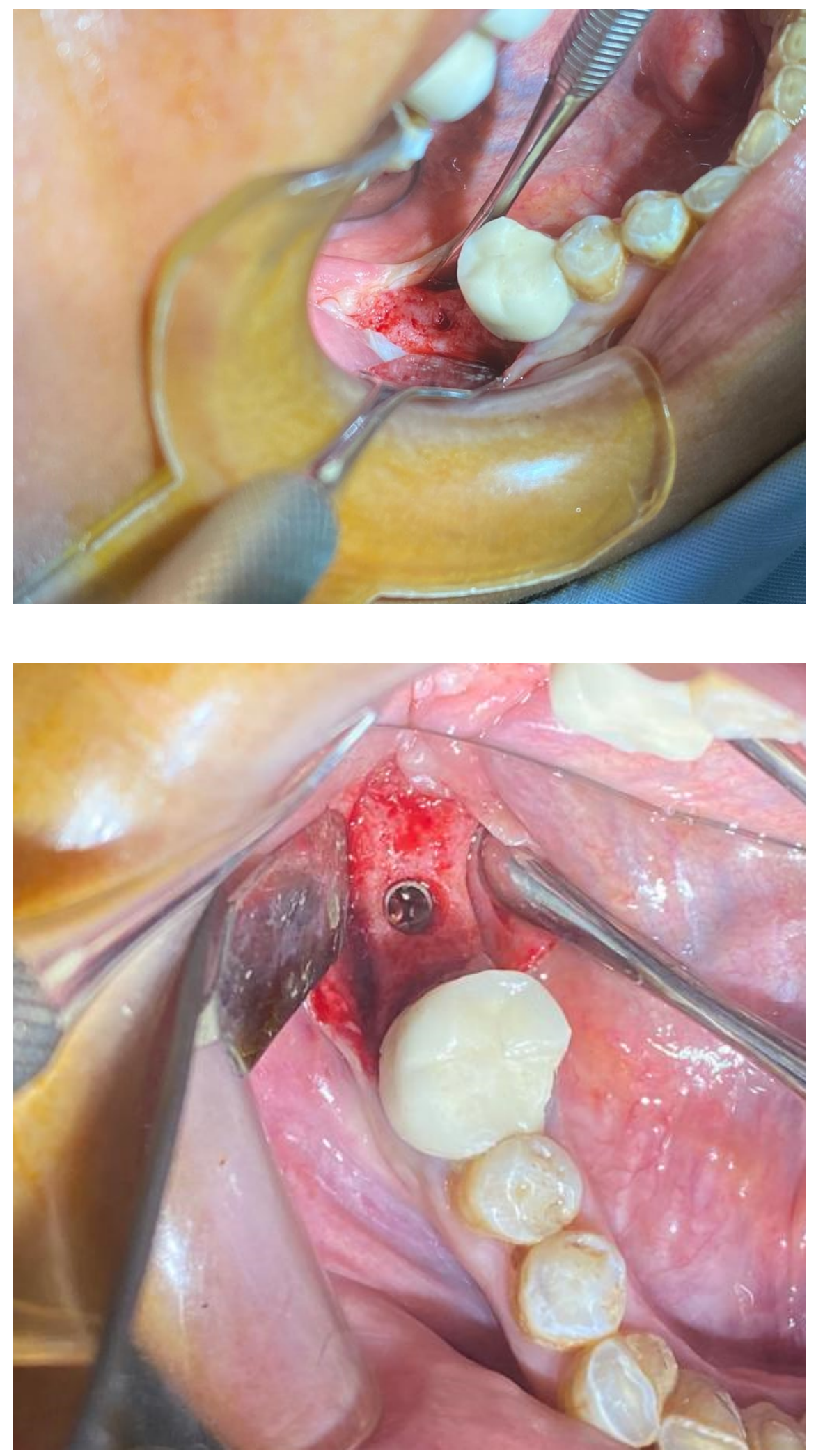


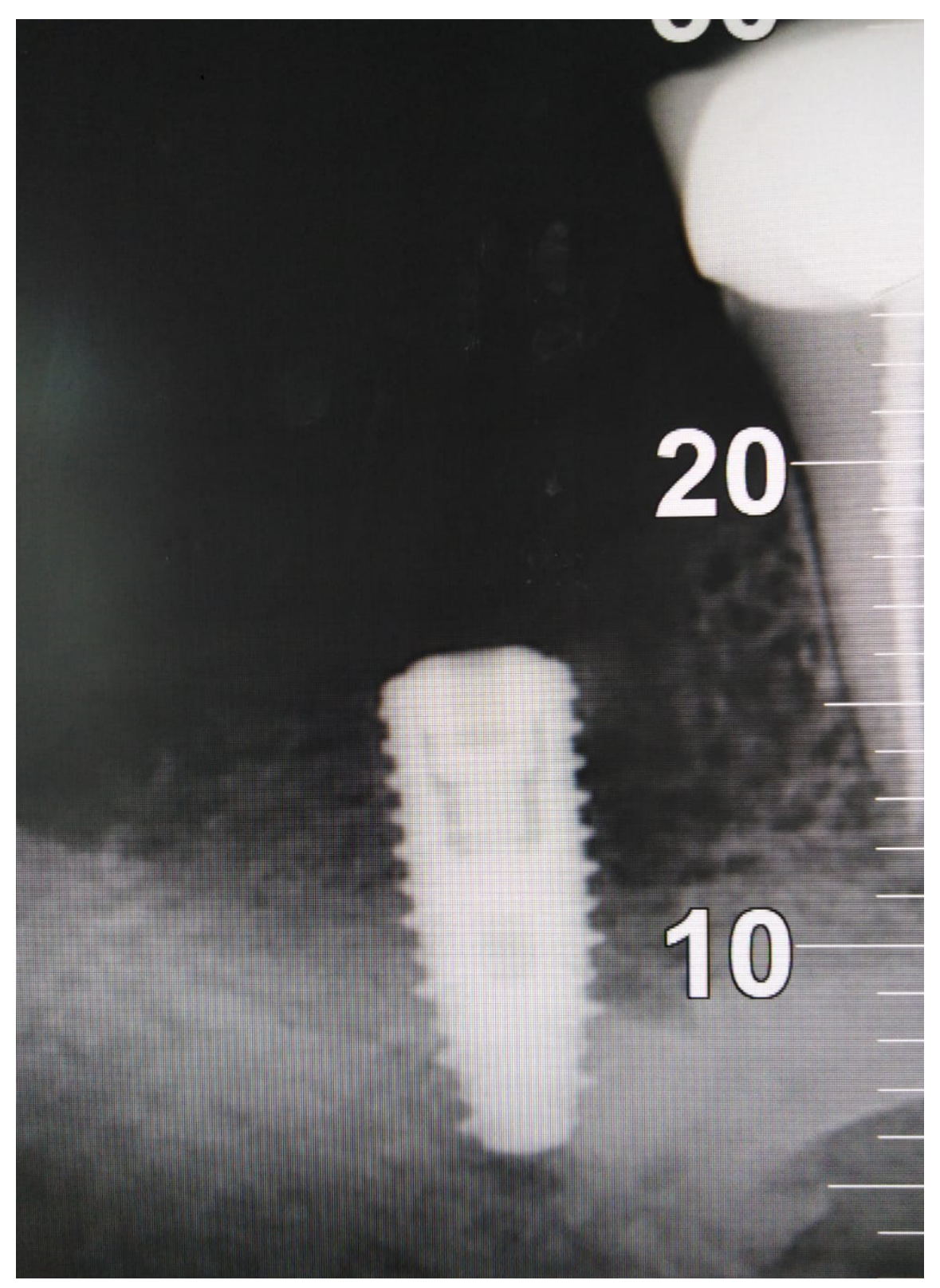



